\title{
A disproportionate contribution of papillary muscles and trabeculations to total left ventricular mass makes choice of cardiovascular magnetic resonance analysis technique critical in Fabry disease
}

\author{
Rebecca Kozor ${ }^{1,2}$, Fraser Callaghan ${ }^{3}$, Michel Tchan ${ }^{4,5}$, Christian Hamilton-Craig ${ }^{6,7}$, Gemma A Figtree ${ }^{1,2^{*}}$ \\ and Stuart M Grieve ${ }^{1,3}$
}

\begin{abstract}
Background: Sphingolipid deposition in Fabry disease causes left ventricular (LV) hypertrophy, of which the accurate assessment is essential. Cardiovascular magnetic resonance (CMR) has been proposed as the gold standard. However, there is debate in the literature as to whether papillary muscles and trabeculations (P\&T) should be included in LV mass (LVM).

Methods/results: We examined the accuracy of 2 CMR methods of assessing LVM and LV volumes, including $\left(M_{\text {inc }} P \& T\right)$ or excluding ( $\left.M_{\text {ex }} P \& T\right) P \& T$, in a cohort of Fabry disease subjects $(n=20)$ compared to a matched control group $(n=20)$. Significant differences between the two measurement methods were observed for LV end-diastolic volume, LV end-systolic volume, LVM, and LV ejection fraction (LVEF) in both groups. These differences were significantly greater in the Fabry group compared to controls, except for LVEF. P\&T contributed to a greater percentage of LVM in Fabry subjects than controls $(20 \pm 1 \%$ vs $13 \pm 2 \%, p=0.01)$. In the control group, both volume-derived methods ( $M_{\text {inc }} P \& T$ or $\left.M_{e x} P \& T\right)$ provided accurate $S V$ measurements compared with the internal reference of velocity-encoded aortic flow. In the Fabry group, inclusion of $P \& T$ ( $M_{\text {inc }} P \& T$ ) resulted in good concordance with phase contrast flow imaging (difference between flow and volume techniques: $1 \pm 3 \mathrm{ml}, p=0.7$ ).

Conclusion: The volumetric contribution of P\&T in Fabry disease is markedly increased relative to healthy controls. Failure to account for this results in significant underestimation of LVM and results in misclassification of a proportion of subjects.
\end{abstract}

Keywords: Fabry disease, Papillary muscles, Left ventricular hypertrophy, Cardiovascular magnetic resonance imaging

\section{Background}

Fabry disease is a X-linked disorder characterized by deficient activity of $\alpha$-galactosidase $\mathrm{A}$, which leads to progressive lysosomal accumulation of complex sphingolipids, predominantly globotriaosylceramide [1]. In the myocardium this typically produces a uniform pattern of left

\footnotetext{
* Correspondence: gemma.figtree@sydney.edu.au

'North Shore Heart Research Group, Kolling Institute of Medical Research, University of Sydney, Sydney, Australia

${ }^{2}$ Department of Cardiology, Royal North Shore Hospital, Sydney, Australia Full list of author information is available at the end of the article
}

ventricular hypertrophy (LVH) that involves the papillary muscles and trabeculations (P\&T), as well as the ventricular walls. Cardiac death is a major contributor to mortality in Fabry disease, and occurs most commonly secondary to arrhythmias and heart failure. LVH and hypertension are the factors that are most associated with cardiac death in Fabry patients [2]. Serial monitoring of left ventricular function (LVF) and left ventricular mass (LVM) in these patients is therefore desirable for both monitoring the progression of disease, and for assessing the response to treatments such as enzyme replacement therapy (ERT). 
Cardiovascular magnetic resonance (CMR) has been proposed as the 'gold standard' non-invasive method of measuring these indices, however, there is debate in the literature as to whether P\&T should be included in the cavity volume or the myocardial mass [3-6]. Figure 1 illustrates these two approaches to ventricular quantification. The Society of Cardiovascular Magnetic Resonance task force on standardized protocols does not currently favor one method over the other, but suggests that inclusion or exclusion of papillary muscles in LVM should be the same as that used in normal reference ranges used for comparison [7].

In this study, we compare two different CMR approaches in quantifying left ventricular volumes, function and mass in a cohort of Fabry disease subjects compared to controls. We hypothesized that incorporating the P\&T during measurements of LVM is of greater importance in patients with ventricular hypertrophy.

\section{Methods}

\section{Study population}

Twenty subjects with known Fabry disease (genotype positive), from the New South Wales and Queensland states Fabry registry databases, who had been referred for CMR as part of clinical management were included. To avoid any influence of gender on our analysis, only male patients were included. Twenty age-matched male controls were randomly selected from a database of 'normal' healthy volunteers with no history of cardiac disease. The study was approved by the relevant Institutional Human Ethics Committees.

\section{Cardiovascular magnetic resonance imaging}

MRI data was acquired using Balanced Steady-State Free Precession (bSSFP) cine imaging on a $1.5 \mathrm{~T}$ Tesla Signa HDxt GE system (TE 1.5 ms; TR 3.4 ms; 20 phases; flip angle $45^{\circ}$; acquisition matrix $224 \times 224$; FOV $35 \mathrm{~cm}$; slice thickness $8 \mathrm{~mm}$; slice gap $2 \mathrm{~mm}$ ) or a 3.0 Tesla GE system (TE: 1.1-1.6 ms; TR: 3.1-3.6 ms; 20 phases; flip angle 40$45^{\circ}$; matrix $256 \times 256$; in-plane resolution $1.4 \mathrm{~mm}$; slice thickness $8 \mathrm{~mm}$; no inter-slice gap). Cardiac chamber volumes and myocardial mass were quantified using a short axis stack of images acquired during end-expiratory breath hold. Phase contrast velocity encoded data was acquired in the ascending aorta at the level of the main pulmonary artery bifurcation using breath-held gradient echo flowencoded cine images acquired with 20 phases (TE: $3.8 \mathrm{~ms}$; TR: 7.0-8.3 ms; matrix $256 \times 256$; in-plane resolution $1.4 \mathrm{~mm}$; slice thickness of $8 \mathrm{~mm}$ ).

\section{Image analysis}

CMR images were viewed using Osirix software (http:// www.osirix-viewer.com) [8]. Analysis was performed using the STIL analysis tool plugin for Osirix (www.stil. net.au/downloads). LV end-diastolic and end-systolic volumes, LV mass (LVM), LV stroke volume (SV) and LV ejection fraction (LVEF) were obtained from the short axis stack by manually contouring end-diastolic and end-systolic endocardial borders and end-diastolic epicardial borders from the base to the apex. The 4chamber and LVLA views were used to confirm the segmentation to ensure accuracy at the base [9]. The LV endocardial border was defined using the two methods described below and depicted in Figure 1:

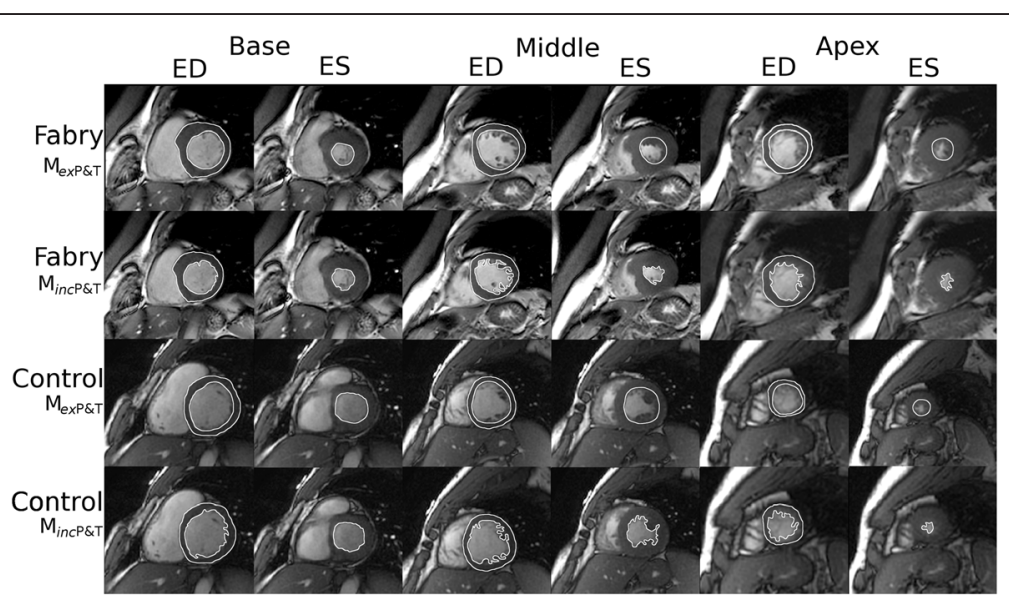

Figure 1 Illustrative example of ventricular assessment. The top two rows show data from an example Fabry subject, and the bottom two show data from an example control subject. For each cohort, the top row panels show epicardial and endocardial contours drawn using Method 2 ( $\left.\mathrm{ex}_{\mathrm{P}} \mathrm{P} \& \mathrm{~T}\right)$ during end-diastole (columns labelled 'ED') and endocardial contours during end-systole ('ES' columns) for representative short axis views at base, middle and apex levels ('Base', 'Middle' and 'Apex' headings). The bottom row for each example show the corresponding contours drawn using Method $1\left(M_{\text {inc }} P \& T\right)$, which includes the trabeculations and papillary muscles. 
- $\mathrm{M}_{\text {inc }} \mathrm{P} \& \mathrm{~T}-$ Papillary muscles and trabeculations with signal intensity within 1 standard deviation of the myocardial signal measured in the LV free wall were manually outlined and included in the myocardial area. Trabeculations below $1.5 \mathrm{~mm}$ were also not included in the segmentation [6].

- $\mathrm{M}_{\mathrm{ex}} \mathrm{P} \& \mathrm{~T}--$ Papillary muscles and trabeculations were excluded from the myocardial area and included in the blood pool by defining the endocardial border as a continuous contour following a smooth path along the compacted myocardium, not including the distinct trabeculations and papillary muscles.

All endocardial and epicardial borders were contoured manually by both an experienced CMR Radiologist (SMG) and Cardiologist (RK). Both endocardial and epicardial borders were traced at end-diastole and only the endocardial borders at end-systole. End-diastole was defined visually as the phase with the largest intracavity volume, and end-systole as the phase with the smallest intracavity volume. The basal slice was selected when at least fifty percent of the left ventricular cavity was surrounded by myocardium at end-diastole. The apical slice was selected as the last frame showing intracavity blood pool at end-diastole. LV volumes and mass were compared between the 2 methods described above in both Fabry and control subjects. As an internal reference, SV calculated using the two methods was then compared with aortic SV measured by velocity-encoded flow sequences in the ascending aorta. Many sites that employ a $\mathrm{M}_{\mathrm{ex}} \mathrm{P} \& \mathrm{~T}$ approach match the LVM calculated from end-systole with the end-diastolic LVM (as a form of internal reference to ensure the same volume of P\&T muscle was included in the blood pool at both end-systole and end-disatole). We chose not to perform this step, as this was felt unnecessary for the purposes of our comparison. Aortic outflow was calculated off-line and manually on a workstation (ReportCARD, GE Healthcare, Milwaukee, WI).

To verify the reproducibility and reliability of the different methods, the intra-observer and inter-observer repeatability of LVEDV and LVESV measurements were assessed in half of the subjects. For intra-observer variability, the measurements were performed twice by the same observer (RK) with at least 1 week between measurements. For inter-observer variability, the first measurements from observer 1 were compared to the measurements calculated by a second independent observer (SG), who was blinded to the initial results.

\section{Statistical analysis}

Statistical analyses were carried out using SPSS 21 (IBM, Armonk, NY). All continuous variables are expressed as mean \pm standard error of the mean. Categorical variables are expressed as frequencies or percentages. Outcome variables were compared using paired-samples t-test for matched variables within each subject group, and independent-samples t-test for variables between the groups. A p-value of $<0.05$ was considered statistically significant. The Bland Altman statistical test was used to assess intra- and inter-observer variability, with the results presented graphically including mean differences and $95 \%$ limits of agreement, and the coefficient of repeatability.

\section{Results}

\section{Participant characteristics}

All participants were males. The Fabry and control groups had a mean age of $42 \pm 3$ vs $35 \pm 1$ years ( $\mathrm{p}=$ 0.08 ), and range $13-66$ vs $27-56$ years, respectively.

\section{Cardiovascular measures}

The measures of LV volumes and mass obtained using the two methods $\left(\mathrm{M}_{i n c} \mathrm{P} \& \mathrm{~T}\right.$ and $\left.\mathrm{M}_{e x} \mathrm{P} \& \mathrm{~T}\right)$ are summarized in Table 1. The two measurement methods resulted in significantly different LV end-diastolic volume (LVEDV), LV end-systolic volume (LVESV), LVM (measured in diastole) and LVEF for both groups. However, the choice of method made a more significant difference in the Fabry population. As shown in Figure 2, the differences between $\mathrm{M}_{\text {inc }} \mathrm{P} \& \mathrm{~T}$ and $\mathrm{M}_{e x} \mathrm{P} \& \mathrm{~T}$ methods were markedly greater in the Fabry group compared to the controls for LVEDV, LVESV and LVM.

By including $\mathrm{P} \& \mathrm{~T}$ in the analysis approach $\left(\mathrm{M}_{i n c} \mathrm{P} \& \mathrm{~T}\right)$, the difference in LVM between Fabry subjects and controls subjects was $178 \pm 14$ vs $130 \pm 6 \mathrm{~g}(\mathrm{p}=0.003)$. This pathological difference was less evident when $\mathrm{P} \& \mathrm{~T}$ were excluded $\left(\mathrm{M}_{e x} \mathrm{P} \& \mathrm{~T}\right.$ : $144 \pm 12$ vs $\left.113 \pm 6 \mathrm{~g}, \mathrm{p}=0.03\right)$. This proportional difference appears to be explained by the fact that the papillary muscles and trabeculations contributed to a greater percentage of cardiac mass in Fabry patients than control subjects $(20 \% \pm 1$ vs $13 \pm 2 \%, \mathrm{p}=$ 0.01 ; Figure $3 \mathrm{~A}$ ). Figure $3 \mathrm{~B}$ is an illustration of the relative contribution of the papillary and trabecular volumes to the overall mass in a Fabry patient.

In order to examine which of the two techniques was more accurate, we compared the stroke volume derived from ventricular contour measurements using both methods with the stroke volume as measured by velocityencoded flow sequences in the ascending aorta, which is renowned for its accuracy [10,11]. Results are shown in Figure 4. In the control group, both volumetric methods $\left(\mathrm{M}_{\text {inc }} \mathrm{P} \& \mathrm{~T}\right.$ or $\left.\mathrm{M}_{e x} \mathrm{P} \& \mathrm{~T}\right)$ gave similar values to the stroke volume calculated from the velocity-encoded data $(\mathrm{p}=\mathrm{ns})$. However, in the Fabry group, exclusion of P\&T $\left(\mathrm{M}_{e x} \mathrm{P} \& \mathrm{~T}\right)$ resulted in a significant $12 \%$ overestimation of SV compared to aortic flow technique (overestimation of $11 \pm$ $4 \mathrm{ml} ; \mathrm{p}=0.007$ ). This was corrected by the inclusion of 
Table 1 Comparison of differences between methods ( $M_{\text {inc }} P \& T$ and $\left.M_{e x} P \& T\right)$ in control and Fabry subjects

\begin{tabular}{|c|c|c|c|c|c|c|}
\hline & \multirow[b]{2}{*}{$M($ incP\&T) } & \multicolumn{2}{|c|}{ CONTROL SUBJECTS $(n=20)$} & \multirow[b]{2}{*}{$M($ incP\&T) } & \multicolumn{2}{|c|}{ FABRY SUBJECTS $(n=20)$} \\
\hline & & $\mathrm{M}(\mathrm{exP} \& \mathrm{~T})$ & p-value & & $M(\operatorname{exP} \& T)$ & p-value \\
\hline LVEDV (ml) & $163 \pm 6$ & $179 \pm 6$ & $<0.001$ & $127 \pm 7$ & $160 \pm 8$ & $<0.001$ \\
\hline LVESV (ml) & $54 \pm 4$ & $71 \pm 3$ & $<0.001$ & $34 \pm 2$ & $56 \pm 3$ & $<0.001$ \\
\hline LVSV (ml) & $108 \pm 5$ & $105 \pm 5$ & 0.2 & $103 \pm 7$ & $100 \pm 8$ & 0.3 \\
\hline LVEF (\%) & $67 \pm 1$ & $61 \pm 1$ & $<0.001$ & $73 \pm 1$ & $65 \pm 1$ & $<0.001$ \\
\hline LVM (g) & $130 \pm 6$ & $113 \pm 6$ & $<0.001$ & $178 \pm 14$ & $144 \pm 12$ & $<0.001$ \\
\hline LVM indexed (g/m2) & $66 \pm 3$ & $58 \pm 3$ & $<0.001$ & $98 \pm 8$ & $79 \pm 7$ & $<0.001$ \\
\hline
\end{tabular}

$\angle V E D V=$ left ventricular end-diastolic volume, $L V E S V=$ left ventricular end-systolic volume, $L V S V=$ left ventricular stroke volume, $L V E F=$ left ventricular ejection fraction, $L V M=$ left ventricular mass, $S V=$ stroke volume.

P\&T (difference between flow and volume techniques: $1 \pm$ $3 \mathrm{ml}, \mathrm{p}=0.7$; Figure 4).

\section{Intra- and inter-observer variability}

Assessment of the intra- and inter-observer variability showed acceptable levels of agreement for both $\mathrm{M}_{i n c} \mathrm{P} \& \mathrm{~T}$ and $\mathrm{M}_{e x} \mathrm{P} \& \mathrm{~T}$, using LVEDV and LVESV as example calculations. Figure 5 shows the Bland Altman graphs and coefficients of repeatability.

\section{Discussion}

In this study, we demonstrate that the choice of including or excluding papillary muscles and trabeculations in LV mass measures can result in large differences in the quantification of LV function and mass that is exacerbated in a condition classically associated with cardiac hypertrophy. We show that in patients with Fabry disease, in which sphingolipids are deposited in myocardial tissue, the contribution of papillary and trabecular

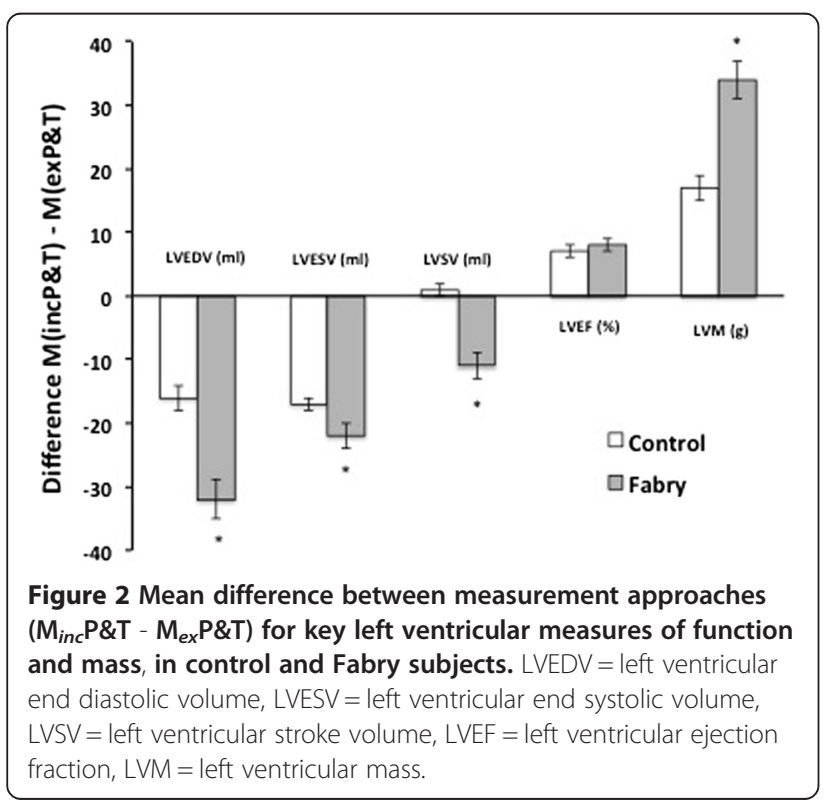

muscle to the total LV mass is markedly increased compared to healthy controls. The inclusion of papillary muscles in myocardial volume improves accuracy of volumetric measures of LV stroke volume when compared with the internal reference measure taken from velocity encoded flow data at the aortic outflow. These findings may have significant implications for assessment and targeted therapy of patients with Fabry disease, as well as other conditions associated with cardiac hypertrophy, and should prompt a move toward more consistent standards in reporting volumetric data.

Our data demonstrate significant differences between the two analysis approaches for both the Fabry and control groups across a range of parameters. While the impact of analysis approach was modest in the control subjects, the magnitude of these differences was much greater in the Fabry cohort (between 10-30\% - see Figure 2). The comparison between the flow-derived LVSV provides strong evidence that $\mathrm{M}_{i n c} \mathrm{P} \& \mathrm{~T}$ is a more accurate approach in the setting of Fabry-related hypertrophy (Figure 4), and even though for control subjects the comparison was non-significant, it is likely that using the $\mathrm{M}_{\text {ex }} \mathrm{P} \& \mathrm{~T}$ approach would introduce a bias toward higher LVEDV, LVESV and LVSV with increasing hypertrophy. In a population setting, this would have the effect of over reporting in these three parameters. We also know, from the Bland Altman plots, that $\mathrm{M}_{\text {inc }} \mathrm{P} \& \mathrm{~T}$ is reproducible and reliable.

Recent data using a computer-aided contouring approach in a large sample $(n=1494)$ drawn from the Framingham study also supports the use of a $\mathrm{M}_{i n c} \mathrm{P} \& \mathrm{~T}$ approach, demonstrating that the inclusion of P\&T in the calculation of LVM results in a measureable difference in normal subjects [12]. The historic motivation for using $\mathrm{M}_{e x} \mathrm{P} \& \mathrm{~T}$ contouring was primarily pragmatic - motivated by the relative speed and reproducibility of this technique in normal subjects using manual contouring. Early computer-based contouring routines were not capable of accurate delineation of trabecular contours, hence the use of this technique has been limited by pragmatic 

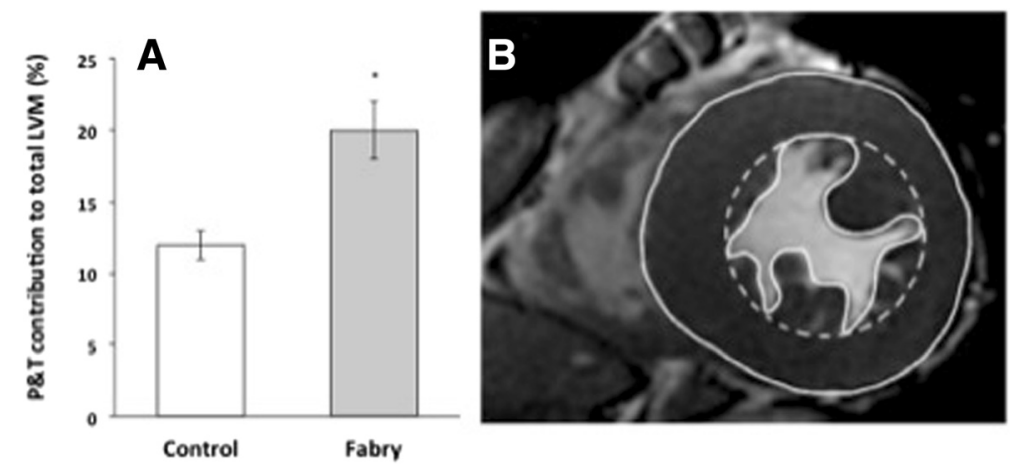

Figure 3 Papillary muscles and trabecular contributions to total LVM. (A) Comparison in control versus Fabry subjects $(p=0.01)$. (B) Graphic showing a mid-ventricular slice illustrating the papillary and trabecular components excluded from the LVM using the Mex P\&T approach (dashed endocardial contour) compared with their inclusion via the MP\&T approach (solid endocardial contour) in a Fabry subject. LVM = left ventricular mass.

considerations [13]. However, in the setting of hypertrophy, the $\mathrm{M}_{e x} \mathrm{P} \& \mathrm{~T}$ approach has difficulties in delineating the interface between tightly packed trabeculae and compacted myocardium without careful attention to detail and close reference to the moving cine images [6]. This is especially difficult towards the apex [12]. This technique is therefore not suited to automation in the presence of hypertrophy due to the increase in compacted trabeculations. Although we use a manual approach in this paper, the increasing availability of computer-aided analysis capable of accurate $\mathrm{M}_{i n c} \mathrm{P} \& \mathrm{~T}$ contours means that the practical advantage of $\mathrm{M}_{e x} \mathrm{P} \& \mathrm{~T}$ is no longer compelling.

In the context of cardiac hypertrophy, the effect of type of volumetric analysis approach on the estimation of cardiac mass is of even greater importance than the functional measures. In the Fabry group, papillary muscle and

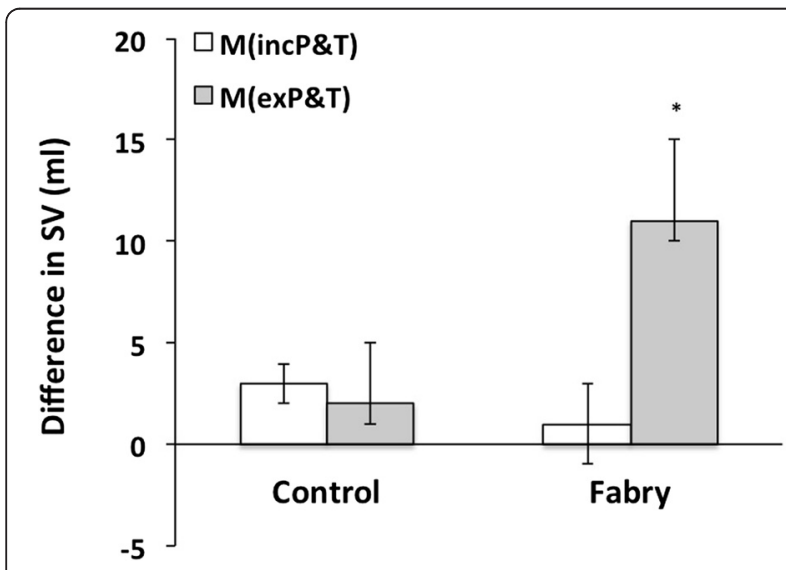

Figure 4 Effect of volumetric analysis method on the determination of LVSV in control versus Fabry subjects. Aortic stroke volume, as measured by velocity-encoded flow sequences in the ascending aorta, was subtracted from LVSV, measured using volumetric methods with both $\mathrm{M}_{\mathrm{inc}} \mathrm{P} \& \mathrm{~T}$ or $\mathrm{M}_{\mathrm{ex}} \mathrm{P} \& \mathrm{~T}$ approaches. LVSV = left ventricular stroke volume. trabecular mass contributed to an average $20 \%$ of the total cardiac mass, over one and a half times more than that of the control group (Figure 3). Thus, comparing to the normal range calculated using a papillary exclusion method (as recommended by different organisations) will not fully account for this, and will therefore underestimate the degree of hypertrophy and cardiac involvement for a patient with Fabry disease. The distortion of this important measure may have implications for patients' disease severity classification and therapy management, which at our institutions is based on organ involvement and the Mainz Severity Scale Index (MSSI) scoring system [14]. Currently, the cardiovascular score in the MSSI includes an assessment of "cardiac muscle thickness" based on electrocardiogram and echocardiography features. In this study, the inclusion of P\&T in LVM measurements increased the number of Fabry patients exceeding the upper limit of normal from $6 / 20$ to $11 / 20$ after normalization by BSA [15]. Therefore, an additional five Fabry patients were reclassified as having an abnormally high cardiac mass using the $\mathrm{M}_{i n c} \mathrm{P} \& \mathrm{~T}$ approach. This effect is driven by the increased contribution papillary muscle hypertrophy makes to overall cardiac mass in Fabry disease, versus nondeposition forms of LV hypertrophy, and has important implications for disease severity classification and therapy in this disease.

Only males were analysed in this study to avoid any influence of gender on hypertrophy in general. We acknowledge that this is a limitation considering Fabry disease affects both genders. It is unclear whether the results can be translated to females, given the gender differences in Fabry cardiomyopathy regarding LVH [16].

The findings of our study have considerable importance in the setting of Fabry disease due to the availability of enzyme replacement therapy (ERT) and the targeting of this therapy to those with identified end-organ involvement. ERT has been available since 2001, and data 


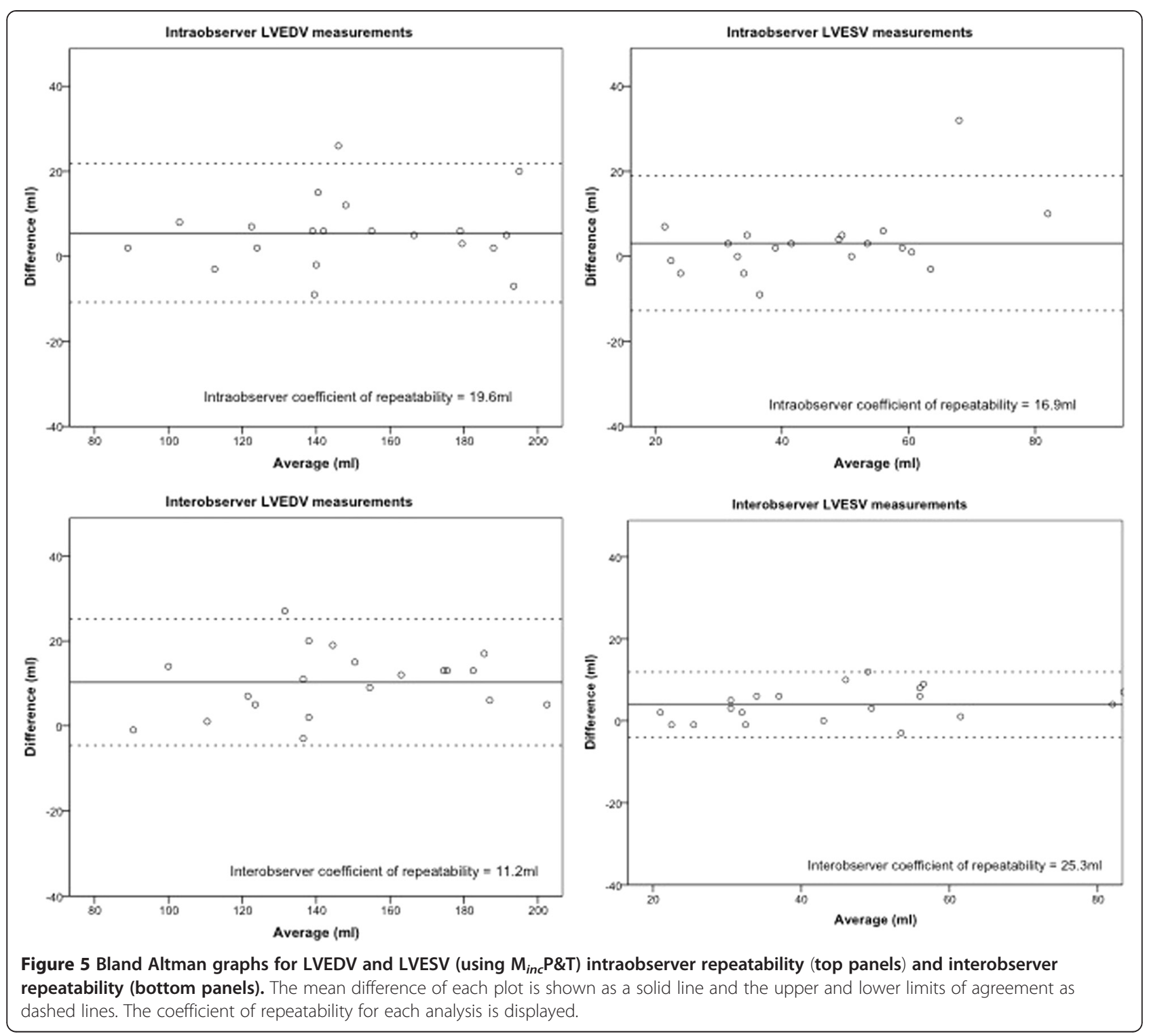

over the last decade suggests that ERT has the potential to change the prognosis of Fabry disease [17-21]. Despite this, there remain numerous uncertainties regarding these agents - these relate to the optimal stage for treatment initiation, considerable heterogeneity of response, and the magnitude of the long-term clinical benefits. The current criteria for initiation of ERT are variable by location, but in Australia include left ventricular hypertrophy as measured by echocardiography or cardiac MRI, or significant arrhythmia. Making use of the $\mathrm{M}_{\text {inc }} \mathrm{P} \& \mathrm{~T}$ approach in this cohort may have altered the eligibility for ERT for five of this study's patients. Our data strongly suggest that accurate measurement of LVM is best achieved using a method that accurately defines the endocardial border so as to include all significant trabeculations and papillary muscles. This analysis deliberately focused on examining subjects with Fabry disease versus controls; further studies are required to explore if the results apply to other types of cardiac hypertrophy.

\section{Conclusion}

The volumetric contribution of papillary muscles and trabeculations in Fabry disease is markedly increased relative to healthy controls in males. Failure to account for this results in significant underestimation of LVM and results in misclassification of a proportion of subjects. While Fabry patients represent an uncommon example of hypertrophy, they underline the importance of accurately contouring the endocardial border for quantification of left ventricular assessment. 


\section{Competing interests}

The authors declare that they have no competing interests.

\section{Authors' contributions}

RK- conception \& design, acquisition/analysis/interpretation of data, manuscript writing; FC- manuscript writing; MC- conception, acquisition of data, manuscript writing; CHC- interpretation of data, manuscript writing; GF- conception \& design, analysis/interpretation of data, manuscript writing SG-conception \& design, analysis/interpretation of data, manuscript writing.

\section{Author details}

${ }^{1}$ North Shore Heart Research Group, Kolling Institute of Medical Research, University of Sydney, Sydney, Australia. ${ }^{2}$ Department of Cardiology, Royal North Shore Hospital, Sydney, Australia. ${ }^{3}$ Sydney Translational Imaging Laboratory, Sydney Medical School, University of Sydney, Sydney, Australia. ${ }^{4}$ Department of Genetic Medicine, Westmead Hospital, Sydney, Australia. ${ }^{5}$ Discipline of Genetic Medicine, Sydney Medical School, University of Sydney, Sydney, Australia. ${ }^{6}$ Heart and Lung Institute, The Prince Charles Hospital, Brisbane, Australia. ${ }^{7}$ University of Queensland, Brisbane, Australia.

Received: 5 August 2014 Accepted: 8 January 2015

Published online: 21 February 2015

\section{References}

1. Zarate YA, Hopkin RJ. Fabry's disease. Lancet. 2008;372:1427-35.

2. Patel MR, Cecchi F, Cizmarik M, Kantola I, Linhart A, Nicholls K, et al. Cardiovascular events in patients with fabry disease natural history data from the fabry registry. Journal of the American College of Cardiology. 2011;57:1093-9.

3. Weinsaft JW, Cham MD, Janik M, Min JK, Henschke Cl, Yankelevitz DF, et al. Left ventricular papillary muscles and trabeculae are significant determinants of cardiac MRI volumetric measurements: effects on clinical standards in patients with advanced systolic dysfunction. Int J Cardiol. 2008;126:359-65.

4. Sievers B, Kirchberg S, Bakan A, Franken U, Trappe HJ. Impact of papillary muscles in ventricular volume and ejection fraction assessment by cardiovascular magnetic resonance. J Cardiovasc Magn Reson. 2004:6:9-16.

5. Janik M, Cham MD, Ross MI, Wang Y, Codella N, Min JK, et al. Effects of papillary muscles and trabeculae on left ventricular quantification: increased impact of methodological variability in patients with left ventricular hypertrophy. J Hypertens. 2008;26:1677-85.

6. Papavassiliu T, Kuhl HP, Schroder M, Suselbeck T, Bondarenko O, Bohm CK, et al. Effect of endocardial trabeculae on left ventricular measurements and measurement reproducibility at cardiovascular MR imaging. Radiology. 2005;236:57-64.

7. Kramer CM, Barkhausen J, Flamm SD, Kim RJ, Nagel E. Standardized cardiovascular magnetic resonance imaging (CMR) protocols, society for cardiovascular magnetic resonance: board of trustees task force on standardized protocols. J Cardiovasc Magn Reson. 2008;10:35.

8. Rosset A, Spadola L, Ratib O, Osiri X. An open-source software for navigating in multidimensional DICOM images. Journal of digital imaging : the official journal of the Society for Computer Applications in Radiology. 2004;17:205-16.

9. Childs H, Ma L, Ma M, Clarke J, Cocker M, Green J, et al. Comparison of long and short axis quantification of left ventricular volume parameters by cardiovascular magnetic resonance, with ex-vivo validation. J Cardiovasc Magn Reson. 2011;13:40.

10. Lotz J, Meier C, Leppert A, Galanski M. Cardiovascular flow measurement with phase-contrast MR imaging: basic facts and implementation. Radiographics. 2002;22:651-71.

11. Gelfand EV, Hughes S, Hauser TH, Yeon SB, Goepfert L, Kissinger KV, et al. Severity of mitral and aortic regurgitation as assessed by cardiovascular magnetic resonance: optimizing correlation with Doppler echocardiography. J Cardiovasc Magn Reson. 2006;8:503-7.

12. Chuang ML, Gona P, Hautvast GL, Salton CJ, Blease SJ, Yeon SB, et al. Correlation of trabeculae and papillary muscles with clinical and cardiac characteristics and impact on CMR measures of LV anatomy and function. JACC Cardiovasc Imaging. 2012;5:1115-23.

13. van der Geest RJ, Buller VG, Jansen E, Lamb HJ, Baur LH, van der Wall EE, et al. Comparison between manual and semiautomated analysis of left ventricular volume parameters from short-axis MR images. J Comput Assist Tomogr. 1997;21:756-65.
14. Whybra C, Kampmann C, Krummenauer F, Ries M, Mengel E, Miebach E, et al. The Mainz Severity Score Index: a new instrument for quantifying the Anderson-Fabry disease phenotype, and the response of patients to enzyme replacement therapy. Clin Genet. 2004;65:299-307.

15. Maceira A, Prasad S, Khan M, Pennell D. Normalized Left Ventricular Systolic and Diastolic Function by Steady State Free Precession Cardiovascular Magnetic Resonance. Journal of Cardiovascular Magnetic Resonance. 2006;8:417-26.

16. Niemann M, Herrmann S, Hu K, Breunig F, Strotmann J, Beer M, et al Differences in Fabry cardiomyopathy between female and male patients: consequences for diagnostic assessment. JACC Cardiovasc Imaging. 2011:4:592-601.

17. Mehta A, Beck M, Eyskens F, Feliciani C, Kantola I, Ramaswami U, et al. Fabry disease: a review of current management strategies. QJM : monthly journal of the Association of Physicians. 2010;103:641-59.

18. Weidemann F, Niemann M, Breunig F, Herrmann S, Beer M, Störk S, et al. Long-term effects of enzyme replacement therapy on fabry cardiomyopathy: evidence for a better outcome with early treatment. Circulation. 2009;119:524-9.

19. Weidemann F, Breunig F, Beer M, Sandstede J, Turschner O, Voelker W, et al. Improvement of cardiac function during enzyme replacement therapy in patients with Fabry disease: a prospective strain rate imaging study. Circulation. 2003;108:1299-301.

20. Imbriaco M, Pisani A, Spinelli L, Cuocolo A, Messalli G, Capuano E, et al. Effects of enzyme-replacement therapy in patients with Anderson-Fabry disease: a prospective long-term cardiac magnetic resonance imaging study. Heart (British Cardiac Society). 2009;95:1103-7.

21. Da H, Elliott PM, Shah J, Zuckerman J, Coghlan G, Brookes J, et al. Effects of enzyme replacement therapy on the cardiomyopathy of Anderson-Fabry disease: a randomised, double-blind, placebo-controlled clinical trial of agalsidase alfa. Heart (British Cardiac Society). 2008;94:153-8.

\section{Submit your next manuscript to BioMed Central and take full advantage of:}

- Convenient online submission

- Thorough peer review

- No space constraints or color figure charges

- Immediate publication on acceptance

- Inclusion in PubMed, CAS, Scopus and Google Scholar

- Research which is freely available for redistribution

Submit your manuscript at www.biomedcentral.com/submit
C Biomed Central 